\title{
Fine-grained Appliance Usage and Energy Monitoring through Mobile and Power-line Sensing
}

\author{
Nirmalya Roy ${ }^{\mathrm{a}, *}$, Nilavra Pathak ${ }^{\mathrm{a}}$, Archan Misra ${ }^{\mathrm{b}}$ \\ ${ }^{a}$ Department of Information Systems, University of Maryland Baltimore County \\ ${ }^{b}$ School of Information Systems, Singapore Management University
}

\begin{abstract}
To promote energy-efficient operations in residential and office buildings, non-intrusive load monitoring (NILM) techniques have been proposed to infer the fine-grained power consumption and usage patterns of appliances from power-line measurement data. Fine-grained monitoring of everyday appliances (such as toasters and coffee makers) can not only promote energy-efficient building operations, but also provide unique insights into the context and activities of individuals. Current building-level NILM techniques are unable to identify the consumption characteristics of relatively low-load appliances, whereas smart-plug based solutions incur significant deployment and maintenance costs. In this paper, we investigate an intermediate architecture, where smart circuit breakers provide measurements of aggregate power consumption at room (or section) level granularity. We then investigate techniques to identify the usage and energy consumption of individual appliances from such measurements. We first develop a novel correlation-based approach called CBPA to identify individual appliances based on both their unique transient and steady-state power signatures. While promising, CBPA fails when the set of candidate appliances is too large. To further improve the accuracy of appliance level usage estimation, we then propose a hybrid system called AARPA, which uses mobile sensing to first infer high-level activities of daily living (ADLs), and then uses knowledge of such ADLs to effectively reduce the set of candidate appliances that potentially contribute to the aggregate readings at any point. We evaluate two variants of this algorithm, and show, using real-life data traces gathered from 10 domestic users, that our fusion of mobile and power-line sensing is very promising: it identified all devices that were used in each data trace, and it identified the usage duration and energy consumption of low-load consumer appliances with $\sim 87 \%$ accuracy.
\end{abstract}

Keywords: energy, plug loads, green building, mobile applications

\footnotetext{
* Corresponding author

Email addresses: nroy@umbc.edu (Nirmalya Roy), nilavra1@umbc.edu (Nilavra Pathak), archanm@smu.edu.sg (Archan Misra)
}

Preprint submitted to Elsevier

January 13, 2016

(C) 2016. This manuscript version is made available under the Elsevier user license

http://www.elsevier.com/open-access/userlicense/1.0/ 


\section{Introduction}

There is widespread interest in developing solutions that provide knowledge of the fine-grained usage and power consumption of everyday appliances (such as coffee makers and televisions) in residential buildings. Such interest is primarily driven by recent interest in energy-efficient building operations, especially as empirical evidence suggests that empowering consumers with greater awareness of their energy consumption patterns can result in 5-20\% reduction in electricity usage [25][26]. However, we believe that, besides this energy-related benefit, the ability to precisely capture the usage profile of everyday consumer appliances also provides insight into an individual's context, at a fine granularity that existing approaches (typically based on mobile sensing [44]) simply cannot provide. For example, while past approaches such as [5, 42, 43] can help classify activities such as "making dinner" or "watching TV", appliance monitoring can additionally indicate that the 'toaster was used today' (revealing details about the food items consumed) or 'the specific TV channel watched' [41].

This paper thus explores the technical feasibility of a vision where the sensing capabilities of bodyworn pervasive devices are combined with the power-line sensing of appliance usage to provide significantly greater insight into the daily activities (formally called Activities of Daily Living or ADLs) of individuals, especially in their residential environments. While the empirical investigations carried out in this paper utilize smartphones (that may or may not be always carried around inside a home), an eventual embodiment will likely rely on wearable devices (e.g., smart-watches [45], smart-bracelets [46]) that are now gaining wider market acceptance and that a user will likely wear almost-continuously [47].

The most common technique for capturing appliance usage is Non-Intrusive Load Monitoring (NILM) [8], where a-priori knowledge of the power signatures of individual electrical devices is used to infer the activation and de-activation of such devices from an observation of the aggregate power consumption signal. Present NILM techniques however operate at two extremes. The most commonly deployed solution involves the use of Smart Energy Meters, installed outside the building, which provide only the total sum of power consumed by all the appliances belonging to that house or consumer [24]. Given such an aggregated observation, we can identify only the major loads in the house (e.g., the HVAC unit or the Washer/Dryer) [7], not the myriad "low-load" appliances (e.g., toasters, TVs, etc.). The other extreme involves the use of wireless "Smart Plugs" $[18,23]$, inserted into individual electrical sockets, and thus providing power measurements at much finer, individual appliance-level granularity. The disadvantage, of course, is that this approach requires the provisioning of such smart plugs in every individual power outlet, resulting in fairly steep deployment challenges and increased monitoring cost.

Motivated by these limitations, our research attempts to answer two questions: a) is there a meaningful alternative, in between the extremes of total vs. individual power consumption monitoring, that helps us in our quest to infer individual appliance consumption patterns?, and b) can the sensing capability of pervasive 
mobile/wearable devices be exploited to improve the accuracy of detecting such appliance usage, thereby providing finer-grained context monitoring?

More specifically, we propose and utilize the Smart Circuit Breaker approach: here each room (or possibly multiple rooms) of a residential or office building is equipped with an instrumented circuit breaker (which we have implemented in ongoing work) which helps measure the aggregate room-level granularity [30]. Such circuit-breakers provide, for example, the total power consumed in the kitchen, without necessarily separating out the concurrent use of one or more appliances, such as the fridge, coffee maker, microwave or toaster. The challenge then is to devise an analytics technique that can help disaggregate such measurements to infer the use of even relatively low-power appliances.

In this paper, we first use real-life measurement studies to develop an enhanced Correlation-Based Power Analytics algorithm, called CBPA, that applies correlation over both macroscopic and microscopic power consumption features, to identify the total usage duration, and the total energy consumption, of individual devices, from such circuit-breaker level aggregated data. While CBPA helps to successfully disaggregate room-level power data into individual devices in some practical cases of interest, its accuracy diminishes if the candidate set of possible low-load devices becomes modestly large. Accordingly, we then explore a joint sensor fusion approach, that combines mobile plus power-line sensing data, to first obtain a smaller, filtered set of candidate appliances whose cumulative power consumption is reflected in the reading of the smart circuit breaker. We provide two different variants of this ADL-driven approach, called Activity-Aware Room-level Power Analytics (AARPA), one rule-based and the other probabilistically-weighted, and then use real-world usage traces to establish their efficacy. Our work thus establishes how a joint fusion of mobile-sensing based ADL recognition and room-level power-line consumption data can provide a practical solution that (a) helps capture the energy consumption characteristics of low-load, commonly-used domestic appliances and (b) provides useful additional context about the lifestyle habits and context of an individual.

\section{Key Contributions:}

- Our key contribution lies in the proposed AARPA approach, that jointly fuses the ADL recognition capabilities of pervasive mobile sensing with the practical power-line sensing offered by Smart Circuit Breakers, to reliably identify the usage and energy consumption profile of relatively low-load daily-use appliances (such as toasters, microwave ovens and treadmills). AARPA provides a practical way to achieve such fine-grained discrimination of appliance usage, without incurring the prohibitive operational overheads of per-device power monitoring via Smart Plugs.

- As a secondary contribution, we suggest CBPA, an enhanced power analytics algorithm, that utilizes correlation measures over long-term traces of both microscopic (transient) and macroscopic (longer-term) power consumption features, to identify the use of such low-load daily-use appliances. While CBPA's accuracy is not adequate when applied in isolation, it performs better than prior ap- 
proaches [7, 40], which are based solely on longer-term 'steady-state' power consumption features. More importantly, when used as part of AARPA, CBPA proves very useful in disambiguating appliance use.

- We evaluate the quantitative benefits of AARPA using real-life activity traces from 10 domestic users, collected over several weeks. Our results show that, given normal everyday patterns of domestic living, AARPA can provide very high accuracy in identifying device usage (AARPA identified $100 \%$ of all devices used in our studies), and significantly increase the accuracy (by approx. 45\%) of estimating perdevice energy consumption. This is achieved even under realistic limits of at-home activity recognition (accuracies of $\approx 90 \%$ ). These results demonstrate the viability of the AARPA approach, for both finer-grained context/activity recognition and appliance-level energy consumption monitoring.

The rest of the paper is organized as follows. Section 2 discusses the related work in this area. Section 3 presents the logical steps of our proposed AARPA framework. Section 4 highlights our initial findings on the power signature analysis. Section 5 presents CBPA, a correlation-based power analytics technique. Section 6 describes an appliance-usage based activity model in combination with the correlation-based power signature analysis. Section 7 presents our detailed experimental results on identifying multiple appliances energy consumption footprint based on an TinyOS smart plug testbed along with an Android smartphonebased real activity data traces. Section 8 identifies future research directions and concludes our work.

\section{Related Work}

Buildings are responsible for at least $40 \%$ of energy use in most countries [6], with efficient and usageaware consumption of electricity by households viewed as a major driver for reducing energy consumption. There has been a significant amount of research on greater visibility of such energy consumption in residential and office buildings [22]. Our work touches on several areas starting from context-aware power signature analysis to building energy management based on plug load meters.

Appliance Power Signature Analysis: Non-intrusive load monitoring (NILM) algorithm was initially proposed by Hart [8] for discerning individual appliance power consumption from total power measurements. The initial technique proposed a cluster analysis approach, over a two-dimensional signature space of real and reactive power. However, the data acquisition system required for the obtaining and storing reactive power measurements is costly. The heuristic end-use load profile algorithm proposed in [17] records the occurrence, timing, and magnitude of large spikes in powerline and disaggregates only relatively large loads, e.g., air conditioners, using a 15 mins sampling dataset, which inevitably limits the range of other consumer appliances that could be detected. An appliance-specific decision rule to detect large appliances such as water heaters and refrigerators has been proposed in [7] with an estimated detection accuracy of about $80 \%$; this approach however, requires excessive training. The appliances connected through a socket in household are 
detected by monitoring the electrical noise in a socket for the transient signals [14]. The FFT of the noise was used as a feature, and the system has required training for each appliance and their combinations. This work, however, incurs significant computational expense of capturing and analyzing the transient noise and needs to be trained on each household appliances. More recently, the authors proposed a new method for detection based on switch mode power supplies (SMPS) and electromagnetic interference (EMI) on the power line [16]. There are several other approaches which address the power signature analysis at higher frequency. A voltagecurrent trajectory based load signatures has been proposed along with hierarchical clustering method to classify and construct the taxonomy of the household appliances [35]. Several classification models for device identification and load signature extraction are investigated using harmonic signature analysis with neural networks [36]. A discriminative sparse coding based approach has been proposed for load disaggregation in contrast to above high frequency approaches that usually rely on special purpose sensors [37]. A templatebased matched filtering approach has been proposed for disaggregating water consumption from a single source [48, 49]. Deep neural networks based energy disaggregation has been investigated in [50]. In [53], EMI noise is used to detect the operating states of the appliances, for example, the surface on which vacuum cleaner is being used to improve energy disaggregation. Acoustic noise of appliances have been captured to identify the micro-states of the appliances which help in fine-grained appliance energy enumeration [54].

Green Building Energy Management using Plug Load Meters: A building energy auditing network based on Wireless AC plug-load meter [18] smart plugs has been proposed in [4]. The MIT plug power meter platform provides apparent power measurements for profiling a load over a short or long time scales [12]. A growing interest for building energy monitoring system has been noticed recently in industry as well due to the several startups, such as EnergyHub [1] and Greenbox [2]. A non-intrusive approach that employs machine learning on data collected from infrastructure sensors, such as magnetic sensors, has been proposed to infer fine-grained power usage in home [10]. PowerPedia enables users to identify and compare the consumption of the plug-level domestic appliances through a smart phone app [32].

Smartphone and Sensor based Energy Prediction: An iPhone App called Beware [3] provides the user information on energy consumption of entire home. It can detect the electricity consumption of different devices and notify the user if the devices use more energy than expected. Energy Lens [13] provides deeper real time visibility of plug-load energy consumption in buildings. It uses the mobile phone to provide a consumer with real-time energy analytics. [15] proposed an ad hoc sensor system that can monitor appliance power usage by exploiting multi-sensor fusion and unsupervised machine learning algorithms. In summary, plug-load meter based approaches can easily achieve a detailed device-level energy footprint but for a steep deployment, operating and maintenance costs [33]. Single sensor-based systems are easier to deploy, but usually have the undesirable properties of requiring custom hardware and extensive appliance-specific training [34]. A circuit-level power measurements and probabilistic level-based disaggregation algorithm to 
separate aggregate data of a limited number of devices has been proposed in [39]. Recently [31] proposed a novel energy disaggregation algorithm along with smartphone based appliance-usage labeling techniques to simplify the training process for identifying appliance-specific power signatures. Our approach is synergistic with studies in [5], which employed activity recognition, principally using infrastructural sensors, to estimate the aggregate energy consumption in a smart home environment. In EnergyLens [55], human activity is inferred form energy consumption based on specific electrical appliance usage, usage duration and location information. The system intelligently combines electricity meter data with sensors on smartphones, WiFi radio and microphone to infer which appliance is being used, when and where it is being used in the home, and who is using it. In [56], a lightweight human activity classification has been performed to identify human activities using smartphones and iBeacon sensor. Multi-user energy consumption monitoring and anomaly detection has been proposed in [51] augmenting the users and environmental contextual information. Disaggregated smart meter data has been employed to detect the ADLs and health status of older adults using a log Gaussian Cox process [52]. In contrast, we focus on using mobile sensing for more practical recognition of Activities of Daily Living (ADL), and focus on estimating the energy consumption at a finer, individual appliance-level, granularity.

\section{The Overall AARPA Framework}

We now introduce the overall AARPA framework, whose logical steps are illustrated in Fig. 1. The framework assumes that a load monitoring device is embedded into the Circuit Breakers associated with each individual room (or often, some logical grouping of rooms), such that this circuit breaker can monitor and provide real-time measurements of the total power consumed by all appliances that draw electrical power through this circuit breaker. The AARPA framework then applies independently to each such circuit breaker. The left-hand portion of the figure operates only on power consumption data and is conceptually similar to past work on NILM-based appliance usage-detection techniques. The algorithm works by first requiring the operation of each individual appliance to be profiled (note that this is a one-time "fingerprinting" process and can even utilize manufacturer-supplied device profiles as an alternative), to build up an appliancespecific power signature. Subsequently, during regular operation, we assume that we obtain the total power consumed, for a specific room, by an (unknown) subset of each appliances that have already been profiled.

In the base implementation of our framework, we simply apply our correlation-based technique, called CBPA, over this total power consumption data, using all the appliances in the room as the potential set of candidates. However, as we shall see, even a modestly large set can cause CBPA to perform fairly poorly. This is where the use of ADL recognition via mobile sensing comes in. We monitor the sensors on the mobile device (in our current implementation, we restrict ourselves to Wi-Fi measurements and accelerometer data, but the concept can be generalized to include a broader array of both mobile and infrastructural sensors 


\section{Activity-Aware Room-level Power Analytics (AARPA)}

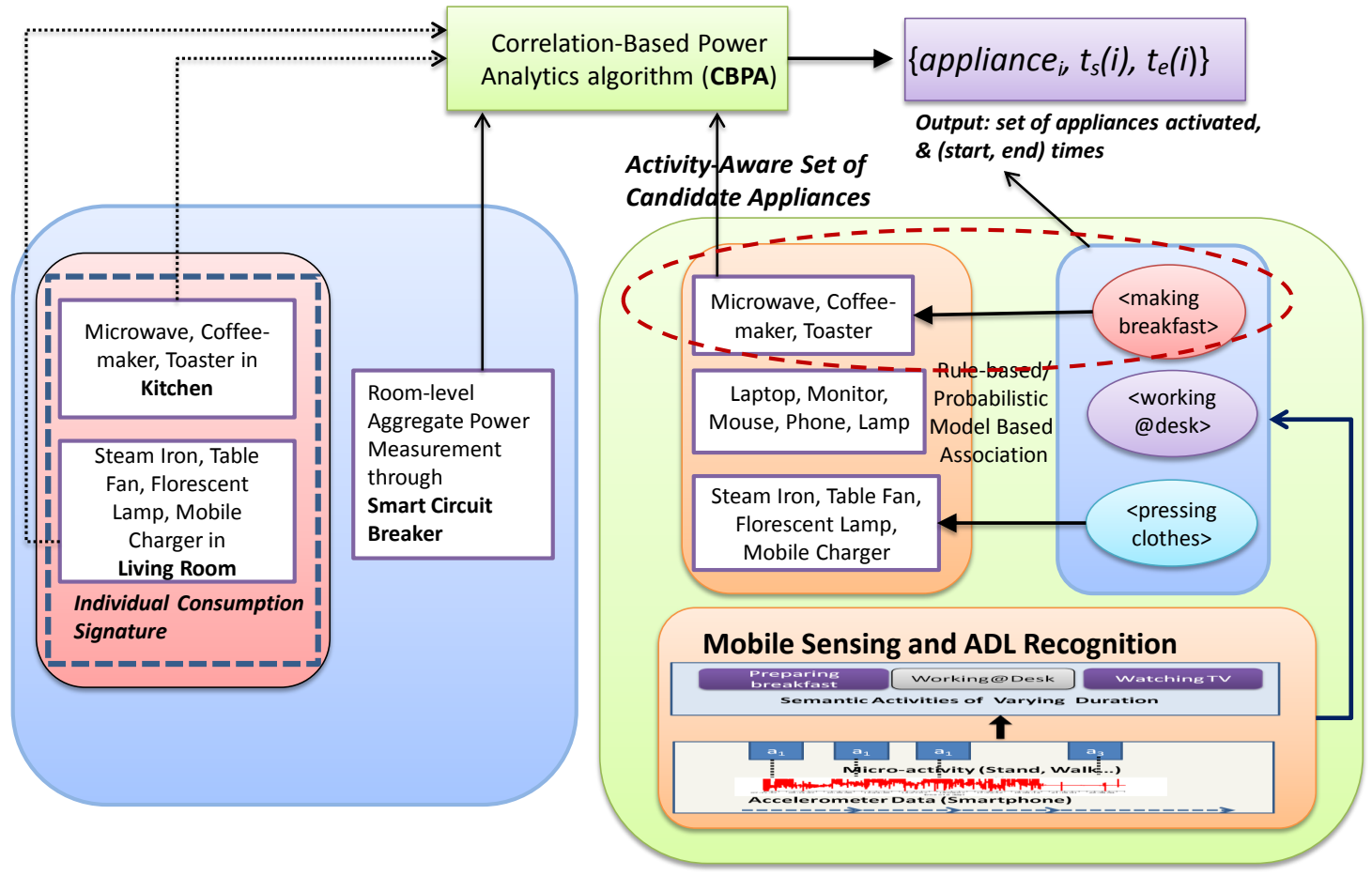

Figure 1: Functional Overview of the AARPA Framework

as shown in [19], and then apply a two-tier activity detection algorithm on this sensor data. In the firsttier, we detect the micro-context of the individual (e.g., the person is walking in the kitchen), and then apply another classifier over this sequence of micro-activities to infer the person's semantic activity-e.g., the "person is preparing dinner". As the next step, this inferred activity is then used to filter down the total set of appliances in the room to a smaller activity-specific set of appliances. (This smaller activity set itself is derived in a supervised fashion from training data, by observing which appliances the user has used during a specific semantic activity.) As the final step in the AARPA approach, the CBPA algorithm is now applied to the measured total power, with this smaller activity-specific set of candidate appliances, to discern the usage pattern and energy consumption of individual appliances.

\section{Appliance Signature Analysis}

Given our focus on identifying relatively low (or medium) load devices, we first present a brief empirical study of the power consumption characteristics of some typical devices. The goal here is to establish that even everyday appliances with seemingly similar power profiles often posses unique temporal characteristics, that we can hope to leverage while attempting to disambiguate the consumption of multiple devices. The absolute values of the instantaneous power readings are not of prime relevance, as: (i) precise readings, 
as well as temporal patterns, will clearly vary across device manufacturers and models, and (ii) individual absolute values are hard to tease out from a power consumption measurement that is a sum of a large number of individual appliances/devices.

More specifically, we study the similarities and differences in the power consumption pattern of two device pairs with relatively quite similar behavior:

1. Refrigerator vs. steam iron: While seemingly quite different in their operation (and role in a consumer's daily life), both exhibit a cyclic pattern of power consumption: the fridge due to duty cycling of its compressor, with the iron due to intermittent deactivation by the thermostat.

2. Table fan vs. fluorescent lamp: While one is a motorized and mechanical device and the one is purely non-mechanical one, both exhibit a relatively steady power consumption pattern when they are on.

Accordingly, our comparison deliberately looks at worst-case scenarios where device pairs have highly similar behavior-other pairings of these 4 devices will be much easier to identify and separate. The measurements are conducted using Moteware Smart Plugs (ACMe) [18], running in a TinyOS environment with an Ubuntu 12.04.3 LTS system (additional details of our measurement experiments will be provided in experimental section).
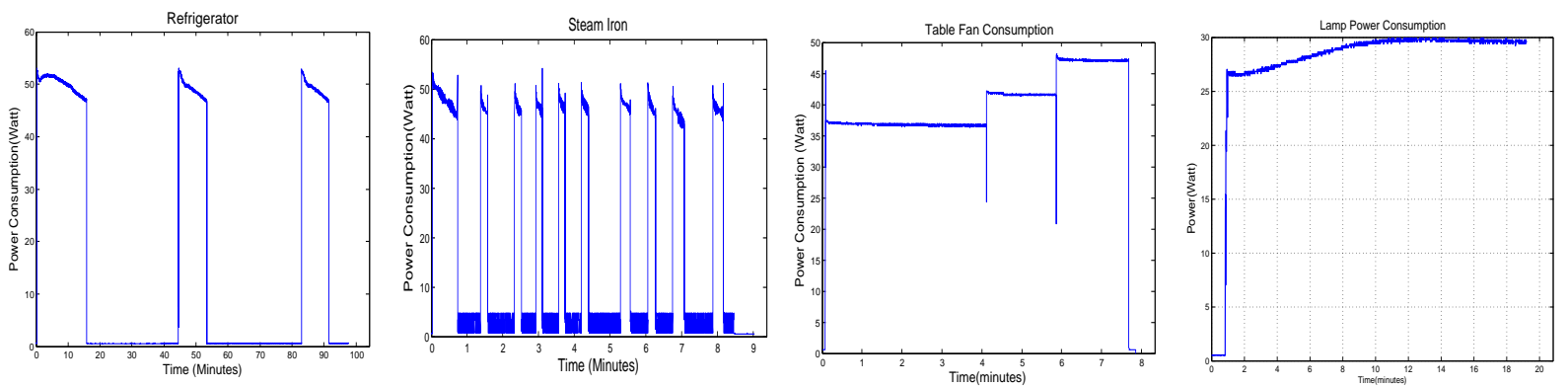

Figure 2: Refrigerator Power Figure 3: Steam Iron Power Figure 4: Table Fan Power Figure 5: Fluorescent Lamp Consumption Consumption Consumption Power Consumption

\subsection{Refrigerator vs. Steam Iron}

Fig. 2 and 3 plots the power consumption pattern of the fridge against that of the steam iron. It should be clear that the consumption behavior exhibits the following similarities: (i) both devices exhibit a very cyclical consumption pattern, with each device being duty cycled for a significant fraction of the time (the fridge having its compressor turned off, while the iron having its heating coil turned off); (ii) both devices having significantly lower (at least 80\%) power consumption during the "off" period of each duty cycle, and (iii) both devices have a longer transient duty cycle (with the fridge's compressor being active to initially reach the pre-set temperature and the iron's heating coil warming up to reach the desired temperature), as they ramp up from an initial state. 
However, a finer-grained inspection of the patterns reveal some clear and insightful differences:

- The time period for the duty cycle is nearly constant for the fridge, while it exhibits irregular variation in the case of the steam iron. However, the "on" period for each duty cycle is fairly constant for both the fridge and the iron. This is really an artefact of the irregular pattern of human usage of the iron, in contrast with the fridge where human interaction is much less frequent and the behavior of the compressor is much more 'regular'.

- Besides the variability, the average value of the time periods are markedly different. The time period for one cycle of the fridge is approx 38-40 minutes, while it is much shorter in the case of the iron (1-1.5 minutes).

- Finally, the power consumption during the "off" period of the duty cycle is quite different-it is essentially about $0.5 \mathrm{~W}$ for the fridge, but around $3 \mathrm{~W}$ for the iron.

The readings suggest that employing a technique that looks not just at initial transients, but over the regular operation cycle of each device, should be able to discriminate between these two devices, by taking advantage of their different temporal evolution patterns.

\subsection{Table Fan vs. Fluorescent Lamp}

Fig. 4 and Fig. 5 plot the power consumption of the table fan and the fluorescent lamp. We can see that both of these eventually have a steady (as opposed to a cyclical) power consumption pattern and that the power consumption 'jumps' to a noticeably large value fairly rapidly. However, more careful inspection also reveals the following key differences:

- While the table fan's transient is nearly negligible (less than 2 secs), the lamp has a pronounced transient phase, taking more than one minute to reach its steady state.

- The readings for the fan show three different "levels". These three levels correspond to the three different speed levels associated with the fan. So, if the consumer actually adjusts the speed level of the fan, the consumption signal will show three different levels (approx $37 \mathrm{~W}, 41 \mathrm{~W}$ and $47 \mathrm{~W}$ ).

Like the previous case, the plots and observations suggest that a temporal pattern matching technique may prove useful in identifying and separate the power consumed by different devices. Note that the measurement plots correspond only to the active power (as the reactive power measurements are not available from the commercial Smart Plug). Had the reactive power readings been available, we would have been able to additionally exploit differences in the amplitude and phase relationship of the reactive component as well. Based on these insights, we now present our proposed CBPA algorithm, which seeks to utilize such temporal signatures in the power consumption characteristics of each individual device. 


\section{Correlation-Based Power Analytics}

The CBPA algorithm proposes to identify both the set of devices/appliances being used, and their individual power consumption, by exploiting both the microscopic and macroscopic features present in the time series of the total power consumption. Microscopic refers to the specific temporal nature of the signal waveforms and harmonics (e.g., as used in [11]), whereas macroscopic refers to power changes, etc as studied in [8]. However, capturing such microscopic features can itself present challenges-for example, to accurately capture harmonics, the Circuit Breaker needs to utilize a higher sampling rate, which in turn poses data transmission bandwidth and storage challenges. Similarly, monitoring the reactive power from the appliances is also a computationally intensive task [11]. To alleviate these problems, [11] proposed to use only transient signals for harmonic analysis. However, we are interested in not just detecting appliances via analyses of their transients, but also estimating their total energy consumption, implying that we need to analyze the steady-state operations as well.

To utilize the signatures present in both transient and steady-state phases of power consumption, our proposed CBPA utilizes a signal waveform analysis technique based on Cross-correlation [9], which captures the similarity of two waveforms as a function of a time-lag applied to one of them. Cross-correlation analysis is often used to detect the presence of a short-duration time series within a longer-duration signal. Mathematically, the cross-correlation (xcorr) sequence, defined between two jointly stationary random processes, $x_{n}$ and $y_{n}$, with $-\infty<n<\infty$, is represented as:

$$
R_{x y}(m)=E\left\{x_{n+m} y_{n}^{*}\right\}=E\left\{x_{n} y_{n-m}^{*}\right\}
$$

where $E\{$.$\} is the expected value operator and asterisk represents the complex conjugation. xcorr (x, y)$ returns the cross-correlation sequence as a vector of size $(2 \times N-1)$ vector, where $\mathrm{x}$ and y are length $N$ vectors $(N>1)$. For continuous-valued signals, xcorr is computed via the convolution of two signals $\mathrm{x}(\mathrm{t})$ and $\mathrm{h}(\mathrm{t})$, by integrating as follows:

$$
y(t)=\int_{-\infty}^{\infty} x(\tau) h(t-\tau) d \tau=x(t) * h(t)
$$

where $\mathrm{y}(\mathrm{t})$ denotes the convoluted signal of two signals. $h(t-\tau)$ represents a $t$ amount time shifted function $h(-\tau)$ assuming the axis is $\tau$. The convolution integral $\mathrm{y}(\mathrm{t})$ is calculated at each time shift $\mathrm{t}$ by multiplying and taking the integration of $x(\tau) \times h(t-\tau) ; \forall \mathrm{t}$ ranging from $-\infty$ to $+\infty . \mathrm{h}(\mathrm{t})$ acts as an impulse response function filter. The convolution procedure is described as follows. (i) reverse the function $h(\tau)$ in time generating $h(-\tau)$, (ii) shift the function $h(\tau)$ by an amount t to have $h(t-\tau)$, (iii) multiply the shifted $h(t-\tau)$ by $x(\tau)$ yielding $x(\tau) h(t-\tau)$, (iv) integrate $x(\tau) h(t-\tau)$ for all positive and negative values of $\mathrm{t}$ considering the overlap to obtain the value of the convolution.

We apply the correlation technique mentioned above to try and identify each individual appliance from the total load measurements. A key aspect of our methodology lies in our modeling and use of both the 
transient and steady-state phases: in particular, peaks in the correlation function often help to identify distinct 'events' associated with each appliance (e.g., a fridge's compressor turning off or on); such peaks correspond to key transient characteristics. However, having isolated these peaks, we then model the steady state load as well to compute the energy consumed over the entire steady-state.

\subsection{Generating Individual Power Signatures}

In our approach, we first individually measure the load behavior (i.e., the characteristic power consumption curve) for each appliance, and thus create a device-specific model or signature of the power consumption. For obtaining the characteristic power consumption of a device or appliance, the device was plugged into an ACMe plug load meter, and monitored for a duration long enough to capture both transient and steady-state phases. We wrote a generic code to collect the relevant characteristic information from these measurements. For example, for the case of a mini-refrigerator, the fridge was left on for at least three compressor ON cycles. In this case, the characteristic information retrieved includes (i) the characteristic energy value when the compressor is ON, (ii) average energy consumption value when the compressor is OFF, (iii) time period of one cycle comprising both successive compressor ON and OFF states, and (iv) the energy consumption during the transient period of the compressor turning OFF.

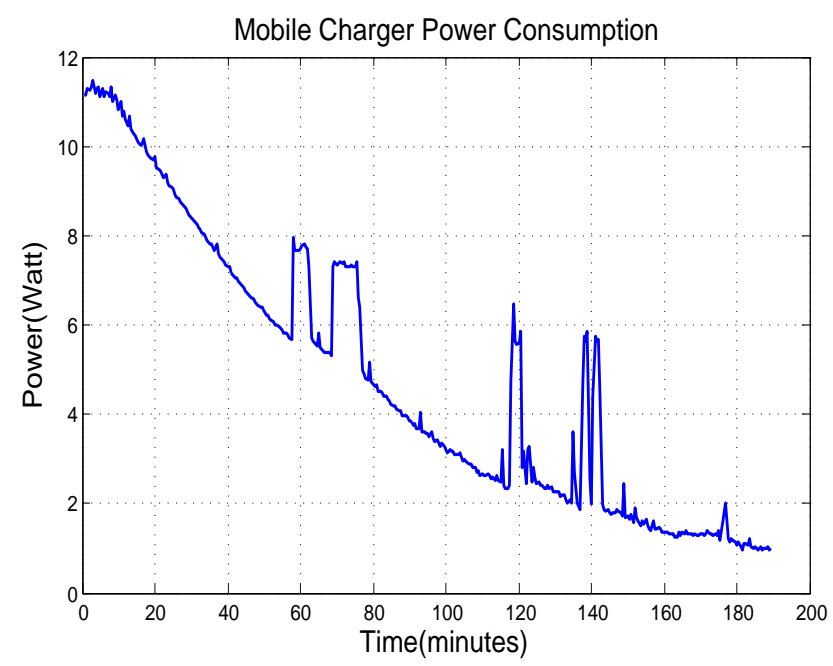

Figure 6: Mobile Charger Load Model

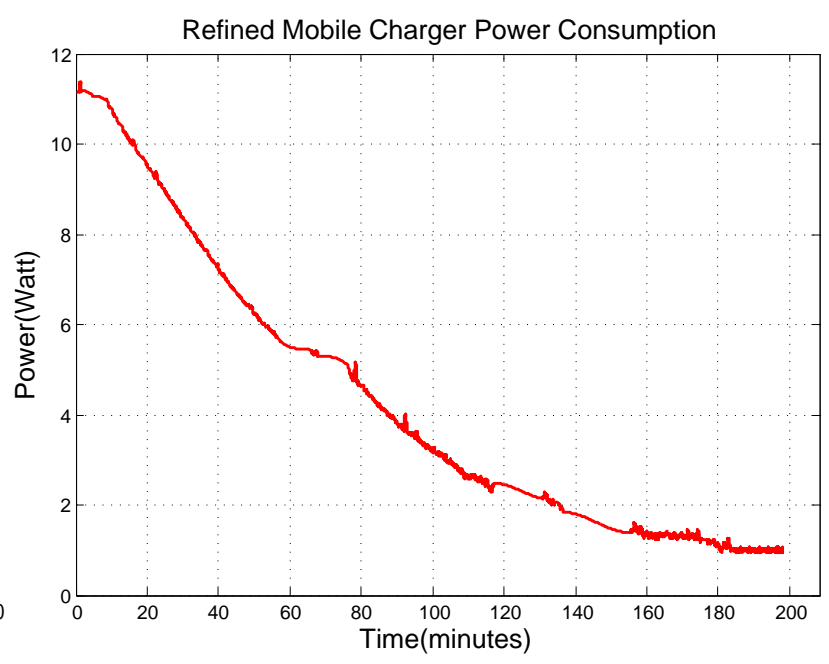

Figure 7: Mobile Charger Characteristic Curve

Similarly, generic codes are implemented for collecting characteristic power curves from other respective devices. To generate accurate characteristic curves, it is important to not only measure for a sufficiently long duration (e.g., to capture the long transient phase observed previously for the case of the fluorescent lamp), but to also avoid usage artefact during the measurement phase. For example, during the power drawn by the Mobile Charger, it is desirable that no phone calls or messages are sent or received. If such communication occurs, the resulting load model will contain peaks (as shown in Fig. 6), as opposed to the exponentially 


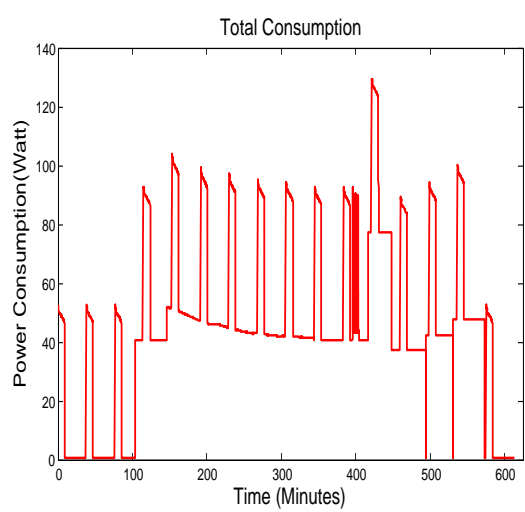

Figure 8: Aggregated Power Consumption

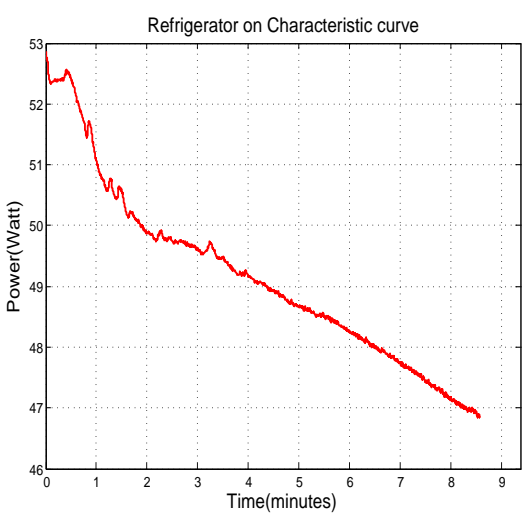

Figure 9: Refrigerator Characteristic

Power Curve

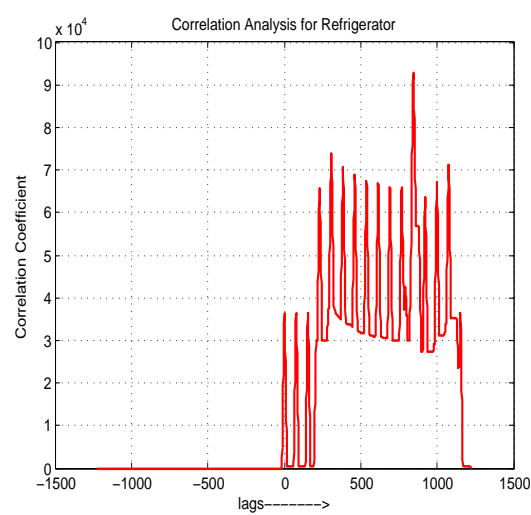

Figure 10: Refrigerator Correlation Analysis Curve

decaying behavior that we expect to see ideally. To overcome such undesired spikes and signal "glitches", our measurement techniques perform signal clipping above a certain threshold and then smoothen out the clipped portion of the curve by using interpolation to approximate missing samples. Fig. 7 represents such a smoothed load modeling curve.

\subsection{Feasibility of the CBPA Approach}

To test the possible use of the correlation-based CBPA approach, we utilize a real dataset collected using the Smart Plug over a 10 hours time period (614 mins) as shown in Fig. 8 from the appliances described below. To generate the ground truth about the device usage, we also collected the precise usage times of each device. a) Mini-Refrigerator is ON from 0 to 614 minutes, b) Fluorescent lamp is ON from 104 to 448 minutes, c) Mobile charger is ON from 145 to 344 minutes, d) Steam Iron is ON from 396 to 440 minutes, e) Table Fan is ON at level 1 speed from 416 to 494 minutes, at level 2 speed from 495 to 531 minutes, at level 3 speed from 532 to 573 minutes.

Fig. 8 plots the aggregate load, as measured by the plug load meter. To evaluate the effectiveness of our proposed CBPA technique, we start by trying to separate out the energy consumptions of refrigerator. The general flow chart of our method is shown in Fig. 11. For this we use correlation between the aggregate data set and characteristic curve generated by one of the load modeling function corresponding to refrigerator. Wherever there is a close enough match of aggregate data set curve (Fig. 8) and refrigerator characteristic power consumption curve (Fig. 9), peaks are generated at those points (Fig. 10). These peak points represent the location wherever refrigerator's Compressor ON state's consumption is present. Accordingly, we simply retrieve the consumption profile from the refrigerator's signature and align the consumption curve to those specific time instances; we then subtract the refrigerator's resulting estimated consumption pattern from the 


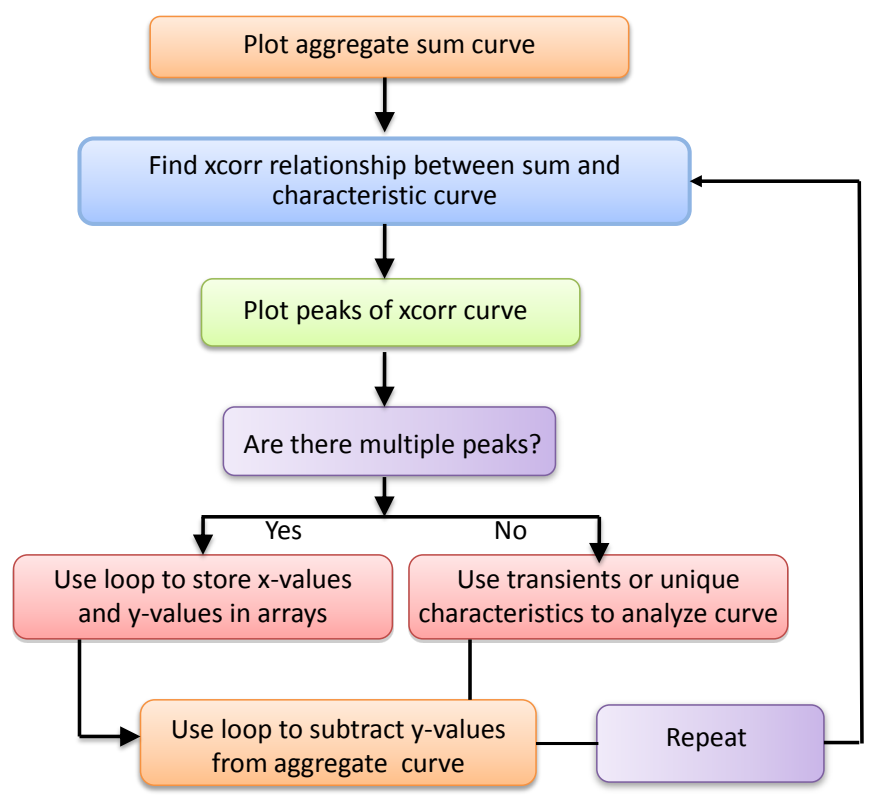

Figure 11: Flowchart of Correlation-Based Power Signature Analysis

total power measurement to get the aggregated consumption of the residual devices, namely the steam iron, the mobile charger, the lamp and the fan. By similarly applying the correlation-based technique iteratively, we recover the energy consumption for both the steam iron and for the mobile telephone charger. Finally, we repeat the process to separate out the consumption patterns of the lamp and the fan. This approach proved to be fairly successful for this somewhat arbitrary mix of appliances, enabling us to recover the energy consumption and usage times for each appliance fairly accurately. As an illustration (to provide a unified view, detailed numerical results are deferred to experimental section), Fig. 12 shows the ground truth total energy consumption excluding the refrigerator, whereas Fig. 13 shows the total energy consumption excluding the $C B P A$-computed energy consumption of the fridge.

While this approach is successful in some cases, it turns out to be incapable of accurately estimating the energy consumption for many other combinations of appliances-in general, larger the set of possible appliances, the poorer the result. Motivated by these empirical observations, we next look into the AARPA approach of first using mobile sensing to infer an individual's ADL context, and using such context to reduce the set of appliances likely being used.

\section{Activity-Aware Room-level Power Analysis}

Our key idea is to leverage upon the large body of work on pervasive/mobile sensing, for inferring both low-level locomotive context [20,21], as well as high-level ADLs [28]. To be clear, this paper does not attempt to innovate in the domain of activity recognition, but instead borrows prior techniques that (i) 


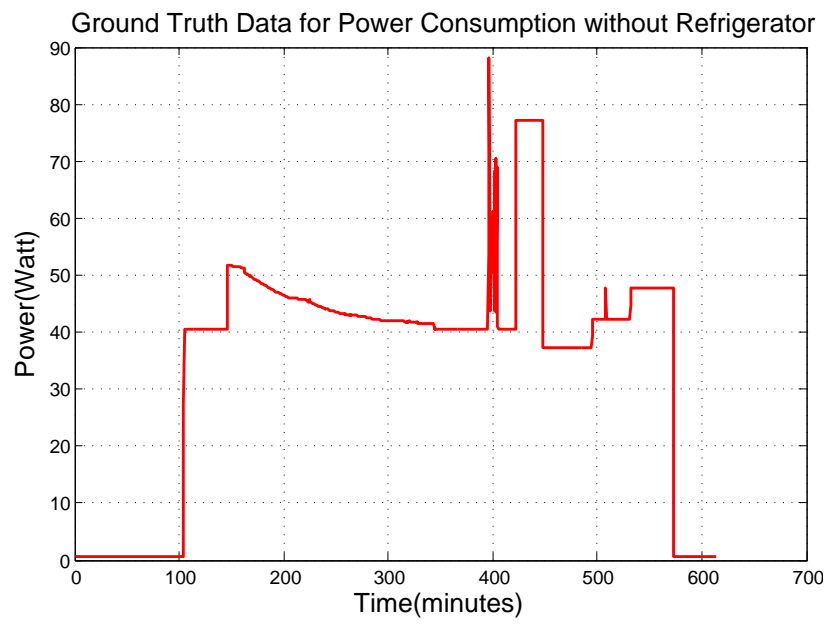

Figure 12: Total Power Consumption excl. Refrigerator (Ground Truth)
Aggregate consumption after Refrigerator consumptions has been removed

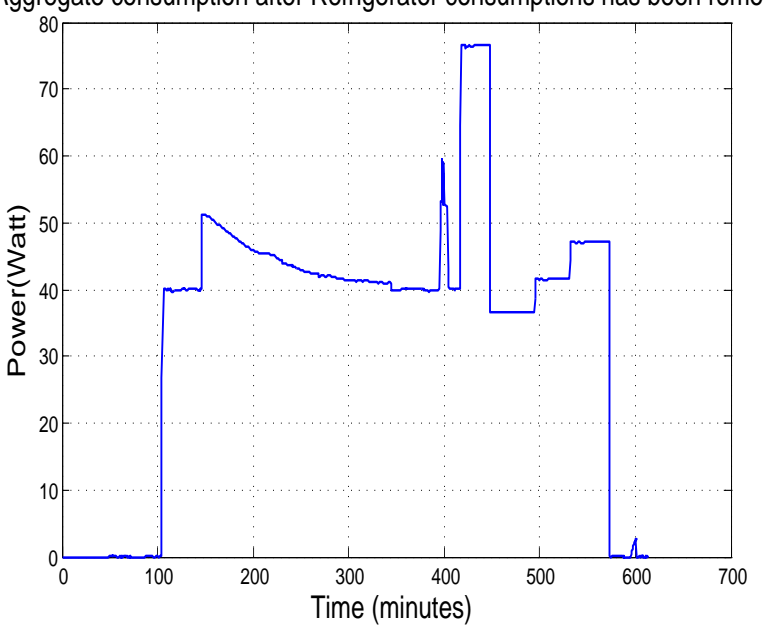

apply a hierarchical classification model [27] to detect various ADLs and (ii) uses Wi-Fi measurement data to determine an individual's location at room-level granularity [29]. Mobile phones themselves have also been used as part of systems for energy attribution in green buildings (e.g., [13]), but more as an information presentation platform, rather than a sensing device.

In particular, for activity recognition studies that we conducted, we assume that an individual carries a smartphone on her body, which provides both an accelerometer sensor stream and a room-level indoor location stream. From the accelerometer data, we first infer a stream of postural states. Consequently, mobile sensing provides us a sequence of (micro-activity, location, timestamp) tuples, where micro-activity refers to an individual's postural state (such as $\{$ walking, sitting, standing, ..., $\}$ ), location refers to the set $\{$ bedroom, bathroom, kitchen,...\}, and time refers to $\{h h: m m: s s A M, h h: m m: s s P M\}$. Subsequently, we extract new features over these tuples (following the SAMMPLE classification approach [27]), and use these features to classify higher-level individual semantic activities (or ADLs), such as $\{$ making breakfast, watching $T V$, ironing clothes, ... $\}$. In general, let $\mathcal{M}$ represent the set of micro-activities and $\mathcal{A}$ represent the set of higher-layer ADLs. (In future, we expect wearable devices such as smart-watches to provide such continuous, and arguably more accurate, sensing in homes and offices.)

\subsection{Appliance-Aware Activity Model}

As the second-stage of the AARPA framework, we then attempt to derive the set of appliances associated with each such ADL. More specifically, we use a-priori training data to associate each element $e, e \in \mathcal{A}$, with a set of appliances that the individual is likely to use (or, more generally, have the appliance be turned on for at least a portion of the overall activity duration) while engaging in that activity. Accordingly, the key 
element of AARPA involves a mapping from each element $e \rightarrow S(e)$, where $S(e)$ is a subset of the overall set of appliances $\mathcal{S}=\{$ microwave, toaster, steamiron, $T V \ldots\}$. Once we have the smaller set $S(e)$, we then apply the CBPA technique described earlier, using this set $S(e)$ and the overall power consumption data obtained from the corresponding Smart Circuit Breaker. In this paper, we propose and explore two-variants of AARPA, that differ in the way the set $S(e)$, for each ADL $e$, is represented and used:

- Rules based: In this approach, called RPA, we collect the total set of appliances used (over the training phase) by an individual when engaging in a specific ADL. More specifically, the set $S(e)$ for any ADL $e$ consists of all the appliances used during any instance of $e$, even if the appliance may have been used only in a small set of such instances, or for a very small duration.

- Usage Weighted: In this approach, called WPA, we compute the probability (or likelihood) $w(e, i)$ of a specific appliance $i$ being used during a specific instance of ADL $e$, by observing and computing the average fraction of time that $i$ is observed to be used during each instance of $e$. In this case, the CBPA algorithm is modified to use not just the absolute maximum correlation value described previously, but instead multiplies the correlation value of each appliance with its corresponding likelihood $w(e, i)$. The WPA technique can be viewed as a Bayesian analogue of RPA, with the identification of a specific appliance being weighted by the a priori likelihood of that appliance being used during a specific ADL.

Fig. 14 provides the high-level pseudocode for the overall AARPA technique, summarizing both the RPA and WPA variants. (For RPA, the $w(e, i)$ values are all set to 1.) Next we shall use empirical investigations to study the benefits and performance characteristics of these two AARPA variants, and compare them with the baseline version of CBPA (which includes in the candidate set any appliance attached to the circuit breaker from which the total power consumption details are obtained).

\section{Experimental Setup and Results}

In this section, we report on our experiments that investigate the benefit of the proposed (AARPA) techniques. Our experiments are conducted with real-life data traces of (i) appliance power consumption and (ii) smartphone sensor readings while participating in ADLs, collected from 10 users living in typical apartments and town home complexes. Table 1 shows the summary of the homes used in our evaluations.

\subsection{Smart Plug Setup and Data Collection}

The Moteware ACMe [18] was used as a plug load meter in this work. The ACMe is based around a Texas Instruments MSP430 microcontroller where power is monitored by an SPI-controlled Analog Devices ADE7753 power monitoring IC, attached with a Hall Effect sensor. The ACMe was plugged into an electrical wall socket; while an individual device was then directly plugged into this plug, an extension cord was used to connect multiple devices. The plug load meter measured and transmitted the power consumption data 
Procedure AARPA (input: aggregate power data $(P)$, output: appliance identification \& power enumeration)

1. Empirically calculate appliance usage based weighting factor from trace data;

1.1 For $(e \in \mathcal{A}) \&(i \in \mathcal{S})\{$

1.1.1 Compute $w(e, i)=\frac{d_{e i}}{\sum_{i=1}^{m} d_{e i}}$ where $w(e, i)$ is the usage weighting factor of $e^{t h}$ activity with

$i^{t h}$ appliance and $d_{e i}$ is the usage time duration of $i^{\text {th }}$ appliance in association with $e^{t h}$ activity.

2. End-For

3. $R P=P ; / /$ residual total power

4. For $(i \in \mathcal{S})\{$

5. Compute correlation $\delta_{i}=\operatorname{xcorr}(R P, i) ; / /$ convolution

6. End-f or

7. Pick the appliance $\hat{i}$ with highest correlation based on $\delta_{i} \times \frac{w(e, i)}{\sqrt{\sum w(e, i)^{2}}}$;

//correlation multiplied by a normalized weighting factor

8. Subtract appliance $\hat{i}$ from $S(e)$ and its characteristic power from $R P / /$ set-theoretic

9. Go to step 7 and repeat the process until $S(e)=\phi$.

Figure 14: The AARPA Algorithm

Table 1: Deployment Logistics

\begin{tabular}{|c|c|c|c|c|}
\hline ID & $\begin{array}{c}\text { Style/Floorplan/ } \\
\text { Built-in-Year }\end{array}$ & $\begin{array}{c}\text { Size (sqft)/ } \\
\text { No. of floors }\end{array}$ & $\begin{array}{c}\text { No. of } \\
\text { Test Apls }\end{array}$ & $\begin{array}{c}\text { No. of Users } \\
\text { Involved }\end{array}$ \\
\hline A1 & Apartment/2-bed-2-bath/1990 & $1100 / 1 \mathrm{flr}$. & 9 & 1 \\
\hline T1 & Townhome/2-bed-1-bath/2001 & $\begin{array}{c}1940 / 2 \mathrm{flrs} . \\
+ \text { basement }\end{array}$ & 15 & 2 \\
\hline A2 & Apartment/2-bed-1-bath/1985 & $1200 / 1 \mathrm{flr}$. & 10 & 1 \\
\hline T2 & Townhome/2-bed-2-bath/1995 & $1300 / 2 \mathrm{flrs}$. & 15 & 2 \\
\hline A3 & Apartment/2-bed-1-bath/1980 & $780 / 2 \mathrm{flrs}$. & 9 & 1 \\
\hline A4 & Apartment/2-bed-1-bath/1974 & $1420 / 1 \mathrm{flr}$. & 10 & 3 \\
\hline
\end{tabular}

in watts (volt-amps), along with a timestamp, at a frequency of $16 \mathrm{~Hz}$, both through a serial and wireless interface to the basestation, which generated a corresponding .csv file for subsequent analysis.

\subsection{Android-based App Development and Data Collection}

We designed an application to collect accelerometer and gyroscope data from an Android based Google Nexus smart phone device for monitoring the activity and appliance usage behavior of a typical user. It also asks the user to manually tag the semantic name of the location (such as bedroom, kitchen, living room etc) and the specific activity being performed to aid in labeling the data, as well as the (start, stop) times of individual appliances. The resulting data was stored in ARFF file format, for subsequent processing using the Weka toolkit [38].

Activities and Data Processing: We collected samples of ten users from six homes as shown in Table 1 performing a variety of both kitchen-related ADLs (such as making breakfast, preparing dinner and washing dishes) and living-room related ADLs (watching TV, etc.). We collected samples for time periods between five to sixty minutes based on a specific activity, with sensor data collected at $80 \mathrm{~Hz}$. While conducting each 
ADL, the users were free to place the smartphone in the on-body position of their choice.

\subsection{Enumeration of Activities}

Consistent with our proposed two-tier architecture [19], the activities we monitored consist of two types:

(i) Low-level (or micro): The set of postural micro-activities consisted of 6 different labels: \{sitting, standing, walking, running, lying, bending .

(ii) High-level (or macro): These consisted of semantically meaningful high-level ADLs associated with a specific room, and included the following 6 labels (the first 3 labels are for the kitchen area and latter 3 labels for living room area):

- Preparing Dinner: Subject prepared dinner using the microwave, oven, and stove;

- Making Breakfast: Subject made breakfast using microwave, coffee-maker and toaster;

- Washing Dishes: Subject washed dishes in Dishwasher;

-Working@Desk: Subject worked at computer desk using laptop, monitor, phone charger, mouse, and lamp;

- Watching TV: Subject watched the TV using TV, DVD player, and a lamp;

- Pressing Clothes: Subject pressed clothes using a steam iron, fluorescent lamp, and table fan with a mini refrigerator and mobile charger on in living room.

Note that each instance of the ADL had definite (start, end) times, manually annotated by each subject. Thus, in this paper, we assume that we have a priori knowledge of the exact mapping between an instance of a macro activity and the underlying set of micro-activities.

Table 2: Confusion Matrix for Appliance Specific Macro Activity

\begin{tabular}{|c|c|c|c|c|c|c|}
\hline Macro-Activity & $\mathrm{a}$ & $\mathrm{b}$ & $\mathrm{c}$ & $\mathrm{d}$ & $\mathrm{e}$ & $\mathrm{f}$ \\
\hline PreparingDinner $=\mathrm{a}$ & 127 & 98 & 0 & 0 & 17 & 0 \\
\hline MakingBreakfast = b & 47 & 458 & 1 & 0 & 25 & 0 \\
\hline Working@Desk = c & 0 & 0 & 486 & 79 & 0 & 19 \\
\hline WatchingTV = d & 0 & 0 & 82 & 522 & 0 & 28 \\
\hline WashingDishes = e & 36 & 81 & 0 & 0 & 91 & 0 \\
\hline PressingClothes = f & 0 & 1 & 45 & 68 & 0 & 136 \\
\hline
\end{tabular}

\subsection{Activity-Aware Power Signature Analysis}

We investigate the issue of whether this activity-aware power signature analytics approach really helps to improve the detection and measurement of multiple appliances' power consumption. In particular, we experimented with 3 different strategies, which differ in whether or not, and how, they use of the additional room-level activity information to reduce the set of candidate appliances used in the CBPA algorithm. 

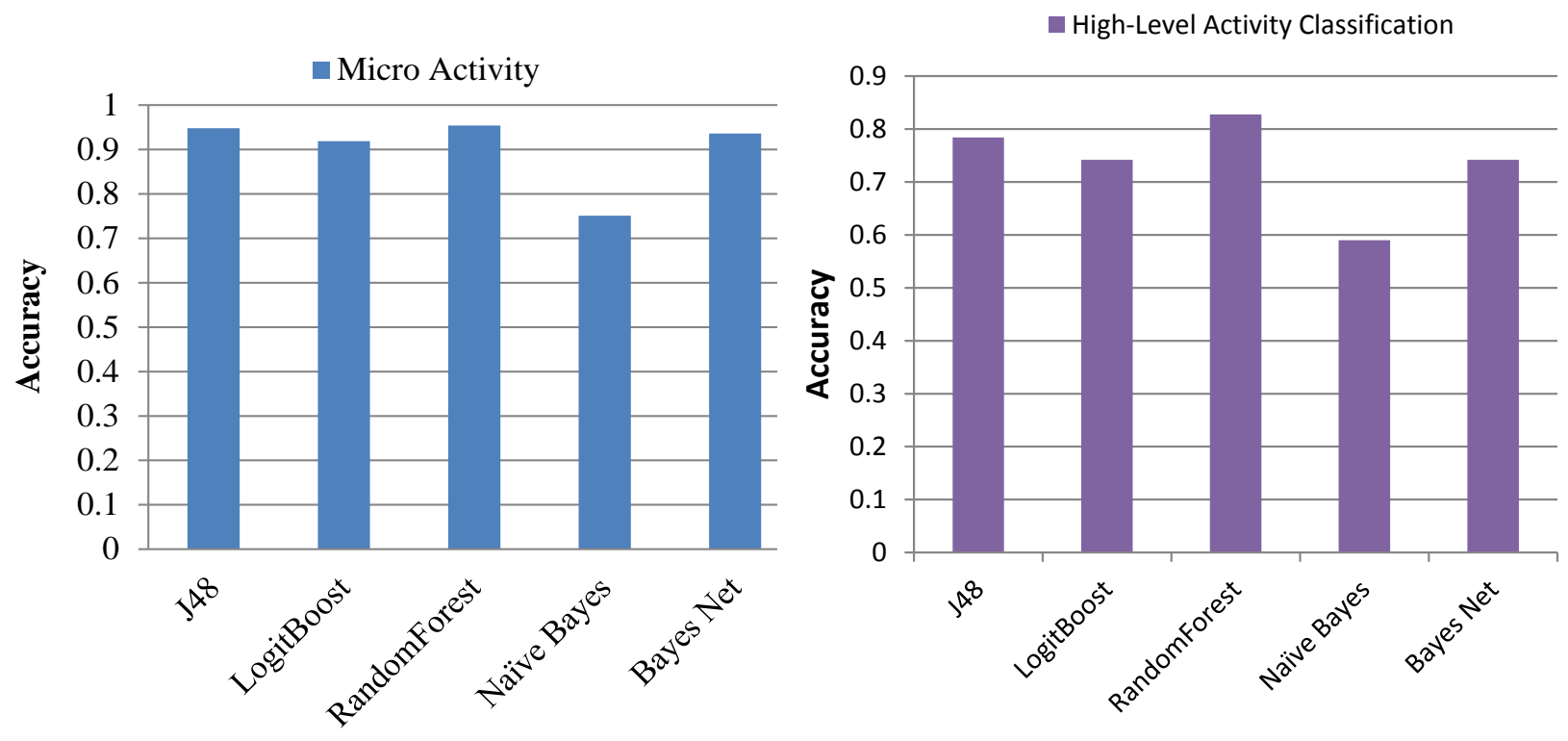

Figure 15: Micro-Activity (bending, sitting, standing, walking, running, lying) Classification Accuracy

Figure 16: Appliance Specific Macro-Activity (Preparing Breakfast, Making Dinner, Washing Dishes, Working@Desk, Watching TV, Pressing Clothes) Classification Accuracy

For each algorithm, we computed both the duration error (the difference between the ground-truth usage duration and that reported by AARPA), and the energy consumption error (the difference between the ground-truth and the AARPA output).

1. Exhaustive Power Analysis (EPA): In this approach, we consider all appliances in a particular location as members of the candidate set, without regard for their use during a specific ADL. For example, for the kitchen area, the set consists of all of the appliances: \{microwave, toaster, coffee maker, boiler, hand mixer, and a grinder\}, even if the user is "making breakfast" and has never used a hand mixer during this ADL. For this set of appliances, we note that the characteristics of the hand mixer were very similar to that of the coffee-maker, making disambiguation via CBPA very difficult. Overall, the EPA approach resulted in fairly high errors (see Table 3 for details), with average (across all appliances) duration and energy consumption errors of $\approx 35 \%$ and $36 \%$ respectively.

2. Rule-based Power Analysis (RPA): In this approach, as explained earlier, the candidate set of appliances was defined a-priori for each separate ADL. For example, using ground-truth data about usage patterns, the "making breakfast" ADL is associated with the smaller appliance set: \{microwave, toaster, coffee maker, and boiler\}, and excludes the \{hand mixer, grinder\} devices. Fig. 15 and Fig. 16 respectively presents the micro and macro activity recognition accuracy using 10-fold cross validation for well known classification algorithms such as J48, LogitBoost, Random Forest, NaiveBayes, and BayesNet. Except for NaiveBayes all other classifiers had similar classification accuracy above $90 \%$. 
A confusion matrix for macro-activity classification using Random Forest algorithm is also shown in

Table 2. The resulting duration and energy consumption errors are lower than that achieved by EPA (about $31 \%$ and $22 \%$ ) respectively.

3. Weighted Power Analysis (WPA): In this weighted-based approach, we additionally compute a usage weight for each candidate appliance (identified by the RPA process), as shown in Line 1.1.1 of Algorithm in Fig. 14, and use this weight to boost the correlation value. In this case, the average errors in duration and energy consumption are sharply reduced, to approx. $12 \%$ and $13 \%$ respectively, attesting to the promise of this approach.

Table 3: Performance Metric

\begin{tabular}{|c|c|c|}
\hline $\begin{array}{c}\text { Power } \\
\text { Analytics } \\
\text { Methods }\end{array}$ & $\begin{array}{c}\text { Start/End } \\
\text { Time } \\
\text { Error (\%) }\end{array}$ & $\begin{array}{c}\text { Total Energy } \\
\text { Consumption } \\
\text { (Joule) Error (\%) }\end{array}$ \\
\hline EPA & 34.78 & 36.17 \\
\hline RPA & 31.25 & 22.12 \\
\hline WPA & 11.74 & 13.27 \\
\hline
\end{tabular}
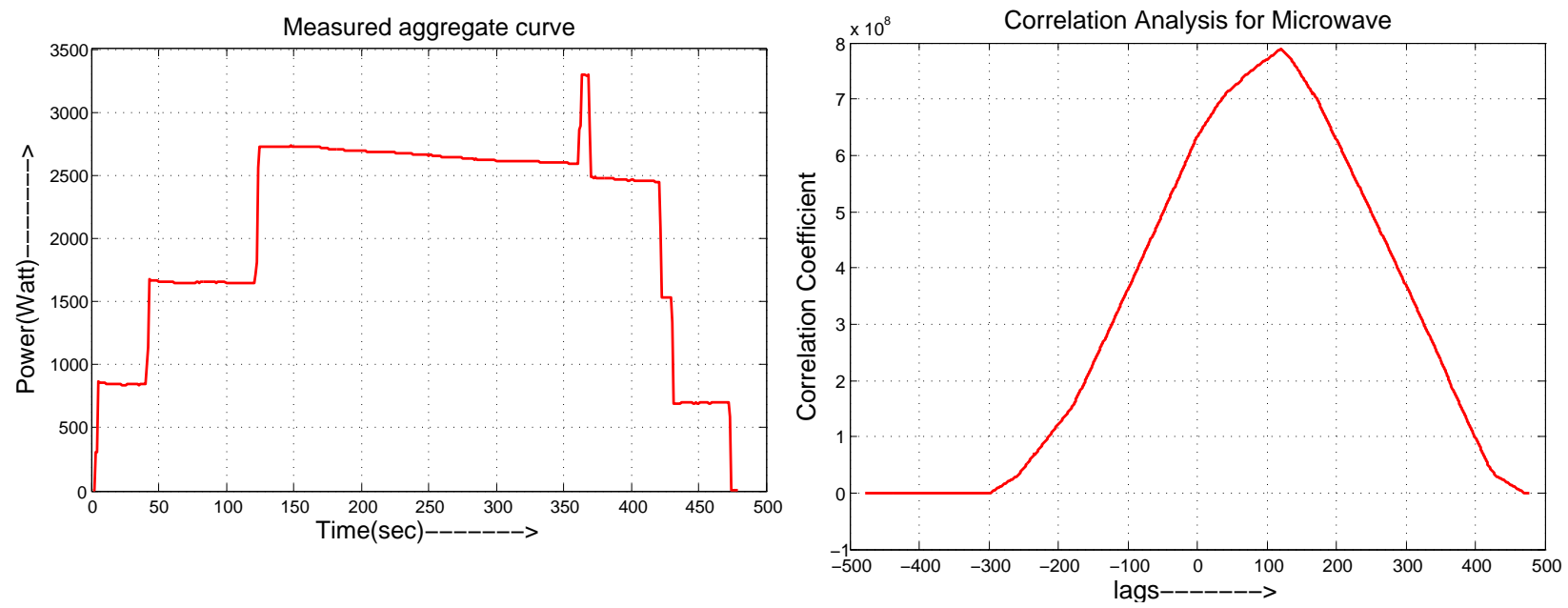

Figure 17: Aggregated Power Consumption Microwave, Coffee Maker, Toaster and Oven

Figure 18: Correlation of Microwave

Fig. 17 represents the aggregate power consumption resulting from the appliances used-namely microwave (121 to $421 \mathrm{sec}$ ), coffee maker (2 to $433 \mathrm{sec}$ ), toaster (360 to $472 \mathrm{sec}$ ) and oven (38 to $372 \mathrm{sec}$ ) associated with macro-activity 'making breakfast'. Fig. 18 presents correlation coefficients multiplied by the weighting factor as calculated at Line 7 in Algorithm 14 for microwave after performing the xcorr on aggregate data. Fig. 19 shows the residual total power consumption without the microwave and Fig. 20 shows the residual total power consumption without the microwave and coffee maker as obtained using WPA technique.

We have also considered the power usage datasets for 3 users for a specific macro-activity 'making 

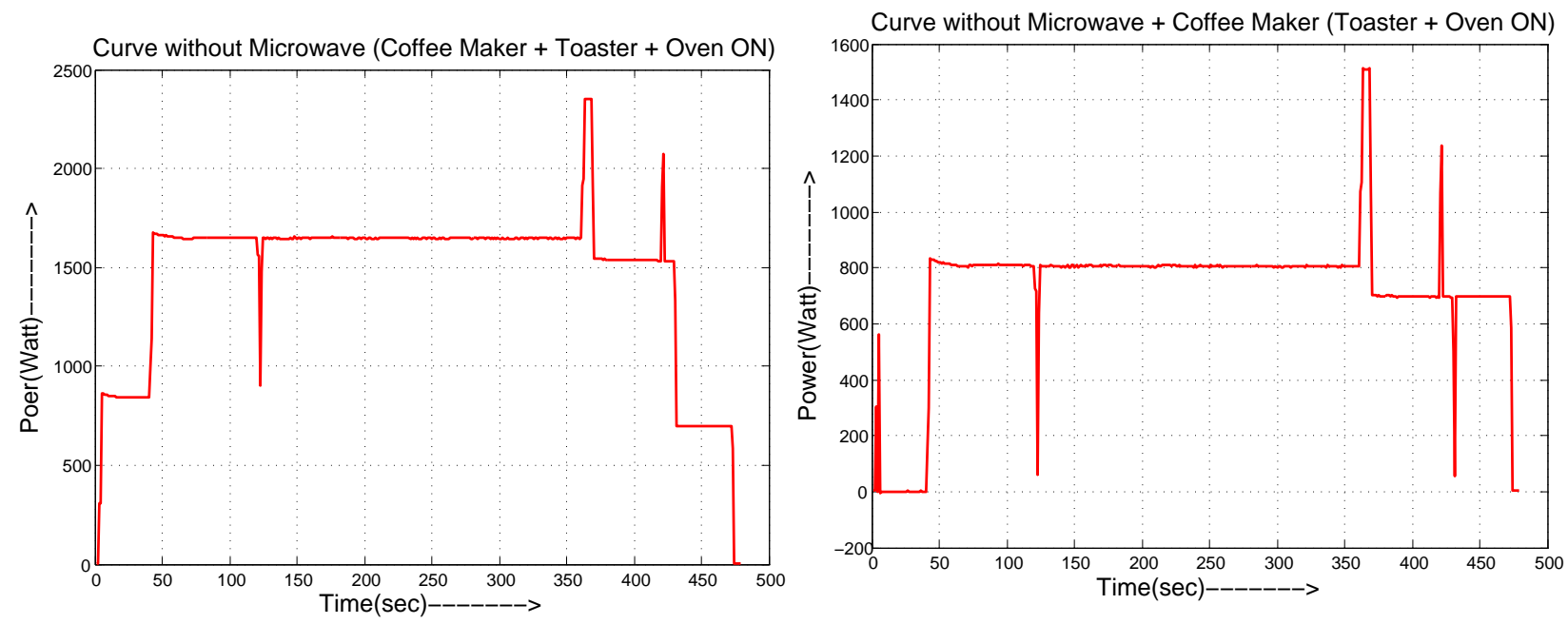

Figure 19: Aggregated Power Consumption Without Microwave

Figure 20: Aggregated Power Consumption Without Microwave, Coffee Maker

breakfast' associated with a set of appliances such as Refrigerator (R), Microwave (M), Coffee-maker (C) and Toaster $(\mathrm{T})$. We have employed our CBPA and AARPA methods on these power consumption traces to find out what specific appliances the users have used for a specific activity. Table 4 (which summarizes the appliance usage and detection accuracies for all 3 users) corroborates that User 1 has made use of all 4 appliances which is detected by CBPA with 75\% and AARPA with a 100\% accuracy. User 2 and User 3 did not make use of toaster and coffee-maker respectively for 'making breakfast' activity, respectively. In this case the usage and non-usage of all the appliances have been detected with a 100\% accuracy by both CBPA and AARPA based methods. Inference of such appliance usage provides deeper insights into the context, habits and preferences of an individual and can help in making contextual recommendations for life style changes (e.g., eating habits).

Table 4: Activity-based Appliance Usage Identification Results

\begin{tabular}{|c|c|c|c|c|c|c|}
\hline $\begin{array}{c}\text { Appliance } \\
\text { Usage Detection } \\
\text { Methods/Users }\end{array}$ & $\begin{array}{c}\mathrm{R} \\
\text { Used }\end{array}$ & $\begin{array}{c}\mathrm{M} \\
\text { Used }\end{array}$ & $\begin{array}{c}\mathrm{C} \\
\text { Used }\end{array}$ & $\begin{array}{c}\mathrm{T} \\
\text { Used }\end{array}$ & $\begin{array}{c}\text { CBPA } \\
\text { Detection } \\
\text { Accuracy }\end{array}$ & $\begin{array}{c}\text { AARPA } \\
\text { Detection } \\
\text { Accuracy }\end{array}$ \\
\hline User 1 & $\checkmark$ & $\checkmark$ & $\checkmark$ & $\checkmark$ & $75 \%$ & $100 \%$ \\
\hline User 2 & $\checkmark$ & $\checkmark$ & $\checkmark$ & $\times$ & $100 \%$ & $100 \%$ \\
\hline User 3 & $\checkmark$ & $\checkmark$ & $\times$ & $\checkmark$ & $100 \%$ & $100 \%$ \\
\hline
\end{tabular}

\subsection{Comparison with Existing Approaches}

Circuit level energy disaggregation algorithms have been proposed comprising two methods - heuristic and Bayesian [39]. A probabilistic Bayesian approach rounding the energy consumption of different appliances to a so called 'steady state' has been considered which either truncated the variability in the power consumption 


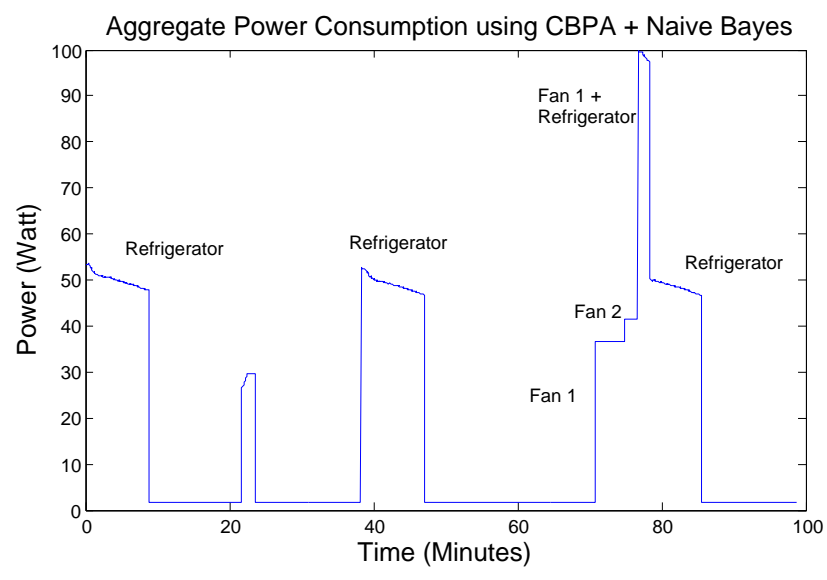

Figure 21: Aggregated Power Consumption using WPA (correlation + probabilistic Bayes)

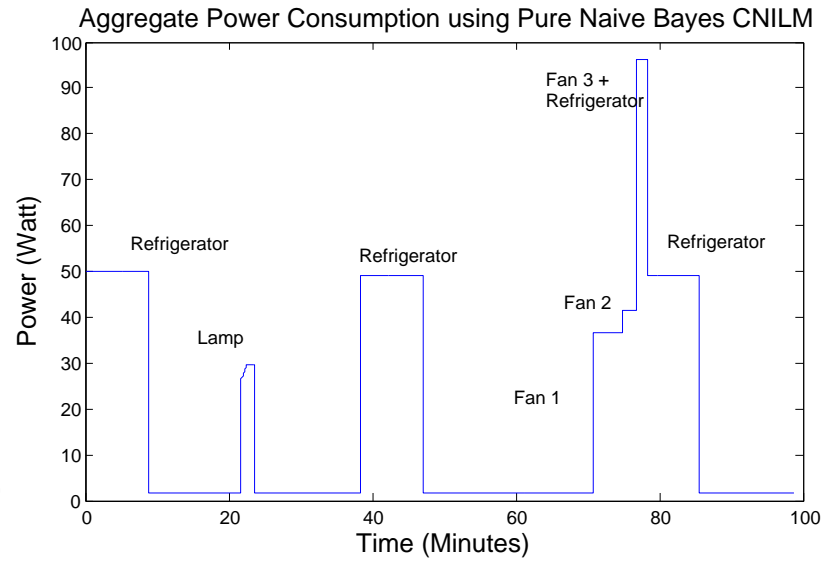

Figure 22: Aggregated Power Consumption using Bayes

of steady-state appliances or ignored the appliances which have periodic nature of their power footprints [40]. Also estimating the probability of a distinct edge by extensive profiling of appliances' changing states is computationally expensive, and as well as error prone given that some of the appliances may have similar edge curves like the refrigerator and steam iron ( $\approx 50$ watts) as shown in Fig. 2 and Fig. 3 respectively. The Bayesian approach of $[39,40]$ was applied on our power consumption traces with a set of appliances (refrigerator (periodic), lamp and fan) and resulted in errors of $\approx 54 \%$, an error rate that is much higher (for this limited study) than our proposed WPA approach. Fig. 21 shows the predicted power consumption curve resulted from applying the correlation and probabilistic Bayesian approach jointly and Fig. 22 represents the predicted power consumption curve where the Bayesian based CNILM (circuit-level non-intrusive load monitoring) method has been employed solely. Table 5 summarizes the performance comparison results of Naive Bayes with respect to our proposed RPA and WPA based methods.

Table 5: Performance Comparison Results

\begin{tabular}{|c|c|c|}
\hline $\begin{array}{c}\text { Power } \\
\text { Analytics } \\
\text { Methods }\end{array}$ & $\begin{array}{c}\text { Start/End } \\
\text { Time } \\
\text { Error (\%) }\end{array}$ & $\begin{array}{c}\text { Total Energy } \\
\text { Consumption } \\
\text { (Joule) Error (\%) }\end{array}$ \\
\hline Naive Bayes CNILM [39, 40] & 8.34 & 53.43 \\
\hline RPA & 7.4 & 16 \\
\hline WPA & 5.47 & 9.8 \\
\hline
\end{tabular}

\section{Conclusion}

In this work, we have advocated an intermediate approach for NILM-based monitoring of both the usage episodes and energy consumption of relatively low-load domestic appliances, that utilizes smart circuit breakers to measure the total power consumption at room (or sections) level granularity. We have developed a 
novel correlation-based analytics algorithm, CBPA, to identify the precise usage duration of, and the overall energy consumed by, appliances such as table fans and coffee makers based on both their steady-state and transient power characteristics. To overcome CBPA's limitations when the set of candidate appliances becomes moderately large, we then propose an analytics approach, called AARPA, that fuses pervasive/mobile sensing and high-level ADL recognition with such circuit breaker-level power readings. AARPA employs mobile sensing to first obtain a reduced set of candidate appliances likely to be used during an ongoing ADL episode, before applying the CBPA technique. Results from a set of 10 users show that a probabilisticallyweighted variation of CBPA shows great promise in identifying the usage of such everyday appliances very accurately (every appliance usage episode was correctly inferred), and provides fairly accurate estimates (average error of around 13\%) of both their usage duration and energy consumption. Future work will explore two challenging aspects: (i) whether additional pervasive sensors (e.g., ambient acoustic sensors) can help improve the accuracy of ADL recognition, thus disambiguating the set of candidate appliances even further; (ii) how AARPA can be extended to work in multi-inhabitant settings, where different users engage in different concurrent ADLs.

\section{Acknowledgement}

The work of Nirmalya Roy is partially supported by the NSF grants CNS-1344990, CNS-1544687, and Constellation $E^{2}$ : Energy to Educate. The work of Archan Misra is partially supported by the Singapore Ministry of Education Academic Research Fund Tier 2 under research grant MOE2011-T21001, and partially by the National Research Foundation, Prime Minister's Ofce, Singapore, under the International Research Centre at Singapore Funding Initiative, and administered by the Interactive \& Digital Media Program Office. We would like to thank Dr. Behrooz Shirazi for his valuable comments and feedback on this work. We would also like to thank the anonymous reviewers for their constructive feedback and valuable comments to help improve the quality of this manuscript.

\section{References}

[1] EnergyHub, http://www.energyhub.com/

[2] Greenbox, http://www.getgreenbox.com/

[3] BeAware. 2010. http://www.energyawareness.eu/beaware/

[4] Xiaofan Jiang, Minh Van Ly, Jay Taneja, Prabal Dutta, and David Culler, "Experiences with a HighFidelity Wireless Building Energy Auditing Network", ACM SenSys conference, Nov. 2009 
[5] C. Chen, B. Das, and D. Cook, "Energy prediction based on resident's activity", Proceedings of the International workshop on Knowledge Discovery from Sensor Data, 2010.

[6] Energy Efficiency in Buildings. 2009. DOI=www.wbcsd.org.

[7] L. Farinaccio, R. Zmeureanu, "Using a pattern recognition approach to disaggregate the total electricity consumption in a house into the major end-uses," Energy and Buildings, vol. 30, pp. 245-259, 1999.

[8] G. W. Hart, "Nonintrusive Appliance Load Monitoring," Proceedings of the IEEE, Vol. 80, pp. 1870-1891, 1992.

[9] Steven Kay, "Fundamentals of Statistical Signal Processing", Prentice Hall

[10] Y. Kim, T. Schmid, Z. M. Charbiwala, and M. B. Srivastava. "Viridiscope: Design and implementation of a fine grained power monitoring system for homes". 11th international conference on Ubiquitous Computing (UbiComp 2009), 2009.

[11] C. Laughman, et al., "Power Signature Analysis," IEEE Power \& Energy Magazine, pp. 56-63, March/April 2003.

[12] J. Lifton, M. Feldmeier, Y. Ono, C. Lewis, and J. A. Paradiso, "A platform for ubiquitous sensor deployment in occupational and domestic environments", IPSN'07: Proceedings of the 6th international conference on Information processing in sensor networks, 2007.

[13] J. J. Ortiz, Y. Noh, G. Saldanha, D. Su, and D. Culler, "Towards Real-time, Finedgrained Energy Analytics in Buildings Through Mobile Phones", In Proc. of 4th ACM Workshop on Embedded Sensing Systems for Energy-Efficiency in Buildings (BuildSys), 2012

[14] S. N. Patel, et al. "At the flick of a switch: Detecting and classifying unique electrical events on the residential power line", In proceedings of UbiComp 2007: 9th International conference. Innsbruck, Austria, 271.

[15] D. E. Phillips et. al, "Supero: A sensor system for unsupervised residential power usage monitoring", IEEE PerCom 2013

[16] S. Gupta, M. S. Reynolds, S. N. Patel, "ElectriSense: Single-Point Sensing Using EMI for Electrical Event Detection and Classification in the Home," Conference on Ubiquitous Computing, pp. 139-148, 2010.

[17] J. Powers, B. Margossian, B. Smith, "Using a Rule-Based Algorithm to Disaggregate End-Use Load Profiles from Premise-Level Data," IEEE Computer Applications in Power, pp. 42-47, 1991. 
[18] ACMe Calibration. Moteware. http://www.moteware.com/acmecal.php

[19] N. Roy, A. Misra, and D. Cook, "Infrastructure-assisted smartphone-based ADL recognition in multiinhabitant smart environments", PerCom 2013: 38-46

[20] J. Kwapisz, G. Weiss, and S. Moore, "Activity recognition using cell phone accelerometers", International Workshop on Knowledge Discovery from Sensor Data, 2010, pp. 10-18.

[21] N. Gyorbiro, A. Fabian, and G. Homanyi, "An activity recognition system for mobile phones", Mobile Networks and Applications, vol. 14, pp. 82-91, 2008.

[22] A. Schoofs, A. Sintoni, and A. G. Ruzelli, "NETBem: Business Equipment Energy Monitoring through Network Auditing," in Proc. of 2nd ACM Workshop on Embedded Sensing Systems for Energy-Efficiency in Buildings (BuildSys), 2010.

[23] Y. Agarwal, B. Balaji, S. Dutta, R. Gupta, and T. Weng, "Managing Plug-Loads for Demand Response within Buildings," in Proc. of 3rd ACM Workshop on Embedded Sensing Systems for Energy-Efficiency in Buildings (BuildSys), 2011.

[24] D. Jung and A. Savvides, "Estimating Building Consumption Breakdowns using ON/OFF State Sensing and Incremental Sub-Meter Deployment," in Proceedings of the 8th ACM Conf. on Embedded Networked Sensor Systems (SenSys), 2010.

[25] M. Chetty, D. Tran, and R. E. Grinter. "Getting to green: understanding resource consumption in the home," In UbiComp'08: Proceedings of the 10th international conference on Ubiquitous computing, pages 242-251, NY, USA, 2008. ACM.

[26] J. Dobson and J. Griffin. Conservation effect of immediate electricity cost. feedback on residential consumption behavior. Proceedings of the 7th ACEEE Summer Study on Energy Efficiency in Buildings, 1992.

[27] Z. Yan, D. Chakraborty, A. Misra, H. Jeung, K. Aberer, "SAMMPLE: Detecting Semantic Indoor Activities in Practical Settings using Locomotive Signatures", International Symposium on Wearable Computers (ISWC), 2012

[28] L. Wang, T. Gu, X. Tao, H. Chen, Jian Lu, "Recognizing multi-user activities using wearable sensors in a smart home", Pervasive and Mobile Computing 7(3): 287-298 (2011)

[29] G.V. Zaruba, M. Huber, F.A. Kamangar, and I. Chlamtac, "Indoor Location Tracking Using RSSI Reading from a Single Access Point", ACM/Springer Journal of Wireless Networks (WINET), vol. 13:2 April 2007. 
[30] Powerwise System, http://www.powerwisesystems.com/

[31] M. Weiss, A. Helfenstein, F. Mattern, and T. Staake, "Leveraging smart meter data to recognize home appliances", IEEE PerCom 2012: 190-197

[32] M. Weiss, T. Staake, F. Mattern and E. Fleisch, "PowerPedia - Changing Energy Usage with the Help of a Community-based Smartphone Application", Journal of Pervasive and Ubiquitous Computing, 2012.

[33] A.G. Ruzzelli, C. Nicolas, A. Schoofs and G.M.P. O'Hare, "Real-Time Recognition and Profiling of Appliances through a Single Electricity Sensor", In Proc. IEEE SECON 2010

[34] M.L. Marceau and R. Zmeureanu, "Nonintrusive load disaggregation computer program to estimate the energy consumption of major end uses in residential buildings", Energy Conversion and Management, 41(1):1389-1403, 2000

[35] H. Lam, G. Fung, and W. Lee, "A Novel Method to Construct Taxonomy Electrical Appliances Based on Load Signatures", IEEE Trans. Consumer Electronics, 53(2):653-660, 2007.

[36] D. Srinivasan, W.S. Ng and A.C. Liew, "Neural-Network-Based Signature Recognition for Harmonic Source Identification", IEEE Transactions on Power Delivery, 21(1):398-405, 2006.

[37] Z. Kolter, S. Batra, and A.Y. Ng, "Energy Disaggregation via Discriminative Sparse Coding", In Proc. Neural Information Processing Systems 2010.

[38] I. Witten, and E. Frank, "Data Mining: Practicial Machine Learning Tools and Techniques with Java Implementations," Morgan Kaufmann, 1999.

[39] A. Marchiori and Q. Han, "Using Circuit-Level Power Measurements in Household Energy Management Systems," Proceedings of the First ACM Workshop on Embedded Sensing Systems for Energy-Efficiency in Buildings (BuildSys), 2009

[40] A. Marchiori, D. Hakkarinen, Q. Han and L. Earle, "Circuit-Level Load Monitoring for Household Energy Management," IEEE Pervasive Computing, Special Issue on Smart Energy Systems, Vol. 10, No. 1, January-March 2011

[41] U. Greveler, B. Justus, "Multimedia Content Identification Through Smart Meter Power Usage Profiles," In Computers, Privacy and Data Protection CPDP, Brussels, Belgium, 2012

[42] S. Phithakkitnukoon et. al., "Activity-Aware Map: Identifying Human Daily Activity Pattern Using Mobile Phone Data," HBU 2010 
[43] D. Cook, A. Crandall, B. Thomas, and N. Krishnan, "CASAS: A smart home in a box," IEEE Computer, 46(6):26-33, 2013

[44] N. Lane, E. Miluzzo, H. Lu, D. Peebles, T. Choudhury, and A. Campbell, "A Survey of Mobile Phone Sensing," Appears in IEEE Communications, September 2010

[45] Android Wear: Information that moves with you, http://googleblog.blogspot.co.uk/2014/03/sharingwhats-up-our-sleeve-android.html

[46] Huawei Smart Bracelet, http://www.huawei.com/us/index.htm

[47] Intel Make it Wearable, https://makeit.intel.com/

[48] D.O. North, "An analysis of the factors which determine signal/noise discrimination in pulsed-carrier systems," Proceedings of the IEEE 51 (7) (1963) 1016-1027

[49] Larson, E., Froehlich, J., Campbell, T., Haggerty, C., Fogarty, J., and Patel, S. N. "Disaggregated Water Sensing from a Single, Pressure-based Sensor: An Extended Analysis of HydroSense using Staged Experiments," The Pervasive and Mobile Computing (PMC) Journal, 2010

[50] J. Kelly and W. Knottenbelt, "Neural NILM: Deep Neural Networks Applied to Energy Disaggregation," ACM BuildSys 2015

[51] P. Arjunan, H. D. Khadilkar, T. Ganu, Z. M. Charbiwala, A. Singh, and P. Singh, "Multi-User Energy Consumption Monitoring and Anomaly Detection with Partial Context Information," ACM BuildSys 2015

[52] J. Alcala, O. Parson, and A. Rogers, "Health Monitoring of Elderly Residents via Disaggregated Smart Meter Data and Log Gaussian Cox Processes," ACM BuildSys 2015

[53] KY Chen, S Gupta, EC Larson, and S Patel, "DOSE: Detecting user-driven operating states of electronic devices from a single sensing point," In Pervasive Computing and Communications (PerCom) March 2015

[54] N. Pathak, MAA. H. Khan, and N. Roy. "Acoustic based appliance state identifications for fine grained energy analytics," In Proceedings of the IEEE International Conference on Pervasive Computing and Communications (PerCom), March 2015

[55] M. Saha, S. Thakur, A. Singh and Y. Agarwal, "EnergyLens: Combining Smartphones with Electricity Meter for Accurate Activity Detection and User Annotation," In Proceedings of ACM E-Energy 2014 
[56] MAU. Alam, N. Pathak, and N. Roy, "Mobeacon: An iBeacon-Assisted smart-phone-Based Real Time Activity Recognition Framework," 12th International Conference on Mobile and Ubiquitous Systems: Computing, Networking and Services, Mobiquitous 2015

Nirmalya Roy is an Assistant Professor in the Department of Information Systems at the University of Maryland, Baltimore County (UMBC). His current research interests include pervasive healthcare, sensordriven health and green technologies, design and modeling of smart environments, and mobile and pervasive systems. He obtained his Ph.D. and M.S. in Computer Science and Engineering from the University of Texas at Arlington in 2008 and 2004 respectively. He received his Bachelors in Computer Science and Engineering from Jadavpur University, India in 2001.

Nilavra Pathak is currently a PhD student in Mobile, Pervasive and Sensor Computing Lab, Information Systems Department at the University of Maryland, Baltimore County. He received his M.E degree in Computer Science and Engineering from Jadavpur University in 2013 and his B. Tech degree in 2011 from St Thomas' College of Engineering and Technology under West Bengal University and Technology in India. His research interests include pervasive computing and energy analytics. He is a member of IEEE.

Archan Misra is an Associate Professor of Information Systems at Singapore Management University (SMU) and a Deputy Director of the joint CMU-SMU Living Analytics Research Center (LARC) with broad research interests in the areas of pervasive computing and mobile systems, including energy-efficient mobile analytics and activity recognition.. Archan received his Ph.D. in Electrical and Computer Engineering from the University of Maryland at College Park in May, 2000, and his B.Tech in Electronics and Communication Engineering from IIT Kharagpur, India in July 1993. 